\title{
Frequency and Time Hopping PPM UWB Multiple Access Communication Scheme
}

\author{
G.S. Biradar, S.N. Merchant, U.B.Desai \\ SPANN Laboratory, Department of Electrical Engineering \\ Indian Institute of Technology Bombay, Mumbai, India \\ Email: \{gsbiradar, merchant, ubdesai\} @ee.iitb.ac.in
}

\begin{abstract}
In this paper we propose frequency and time hopping pulse position modulation (FTH-PPM) ultra wideband (UWB) for multiple access communications. We have derived and investigated the bit error probability for the multi-user synchronous transmitter case in multipath channels with Additive White Gaussian Noise (AWGN). Simulation results show that bit error probability performance of FTH-PPM UWB out performs the time hopping pulse position modulated (TH-PPM) UWB system. It also show that multiuser capacity of FTH-PPM UWB system is much better than TH-PPM UWB system.
\end{abstract}

Index Terms-Frequency hopping, Hybrid hopping, Modulation, Multiple access, Time hopping, UWB.

\section{INTRODUCTION}

Ultra wideband [1] is a new technology that has the potential to revolutionize wireless communication by delivering high data rates with very low power densities. According to the FCC definition, UWB technique is a transmission scheme that occupies a bandwidth of more than $20 \%$ of its centre frequency, or nominally more than $500 \mathrm{MHz}$. The UWB nature offers extensive multipath diversity and support multiple access. The unique characteristics of UWB make it a viable candidate for future wireless communications, especially indoor wireless systems. Several attempts are being made to use UWB as the physical layer for Personal Area Networks (PAN) to meet the FCC standards [2]. FCC has released $3.1 \mathrm{GHz}$ to 10.6 $\mathrm{GHz}$ frequency spectrum with restrictions on minimum transmission bandwidth of $500 \mathrm{MHz}$ and transmit power spectral density of $-41.3 \mathrm{dBm} / \mathrm{MHz}$, which restricts the UWB to be used for PAN. Earlier UWB systems were carrier-free but to utilize FCC's $3.1 \mathrm{GHz}$ to $10.6 \mathrm{GHz}$ band of spectrum UWB system requires the use of a carrier.

In a multiple access scenario, the presence of multiple signals being transmitted at the same time is a typical source of interference for wireless signals. There are several multiple access schemes proposed for UWB, namely, Time Hopping (TH) [3], [4], Frequency Hopping (FH) [5] and Direct Sequence (DS) [6] wherein orthogonal codes are used to avoid multiple access interference (MAI). Efforts have been made to reduce the multiple access interference by designing orthogonal hopping sequences [7]-[9]. However, in practice, the received signal from different users are not orthogonal because of multipath and asynchronous transmission. Moreover, it is not possible to design orthogonal codes for all shifts. In TH, MAI can be reduced by increasing the number of time hops but at the cost of reduced data rate. Frequency Hopping OFDM considered in [10] shows that MAI can be completely removed using Costas sequence under synchronized multiple access communication. Time hopping and frequency hopping is studied separately for enhancing the information rate in [11]. In [12] Time-Frequency hopping multiple access packet communication is considered for studying throughput of ALOHA protocol.

In this paper, we propose a new modulation technique based on frequency and time hopping PPM UWB which greatly reduces the MAI under multiple access communication in comparison with conventional time hopping or frequency hopping schemes. The probability of error is derived for multi-user synchronous transmitter in UWB multipath channel with AWGN. Simulations are carried out for synchronous transmitter case. Second derivative of Gaussian pulse is considered for multiple access analysis.

This paper is organized as follows. In Section II, the system model and construction of the frequency and time hopping PPM UWB signals is described. In Section III, multiple access interference and error probability analysis is presented. Simulation results are discussed in Section IV and concluding remarks are presented in Section V.

\section{SignAL AND SYSTEM MODEL}

The frequency and time hopping M-ary PPM system model for $v$ th user is given by

$s^{v}(t)=\sum_{j=-\infty}^{\infty} A_{d_{\left\lfloor j / N_{s}\right\rfloor}^{(v)}} p\left(t-j T_{f}-c_{j}^{(v)} T_{c}-\delta_{d_{\left\lfloor j / N_{s}\right\rfloor}^{(v)}}\right) e^{-j 2 \pi k_{j}^{(v)} t}$

where, $A^{(v)}$ is the signal amplitude, $p(t)$ represents the second derivative of Gaussian pulse with pulse width $T_{p}$, $T_{f}$ is the frame duration, where a frame is divided into $N_{t h}$ time slots with duration $T_{c}$. The pulse shift pattern $c_{j}^{(v)}, 0 \leq c_{j}^{(v)} \leq N_{t h}\left(N_{t h} T_{c}=T_{f}\right)$ is also called the time hopping sequence for $v$ th source and it is pseudo random with period $T_{c}$. This additional shift avoids catastrophic collisions due to multiple access interference. The sequence $d$ is the data stream generated by the $v$ th source after channel coding and $\delta$ is the additional time shift utilized by M-ary PPM. $N_{s}$ represents repetition 


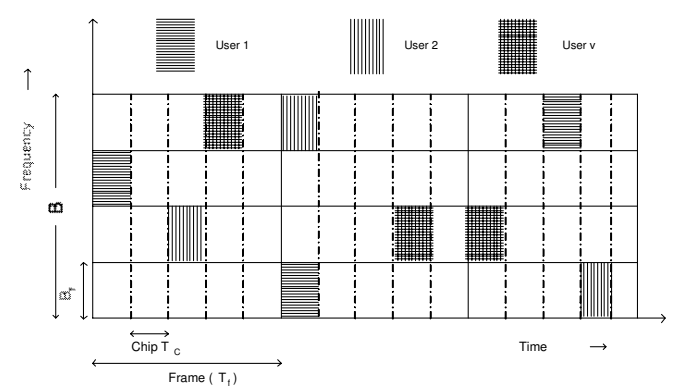

Figure 1. Principle of frequency and time hopping UWB system

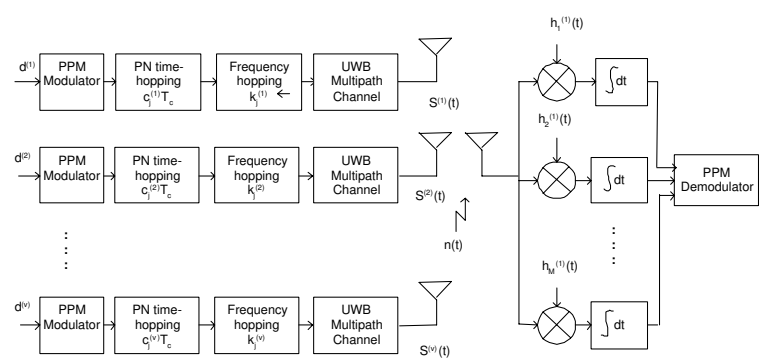

Figure 2. System model of FTH-MA M-ary PPM UWB system

code length with $N_{s}$ pulses being used to transmit the same information. Frequency spectrum is divided into $N_{f h}$ bands with minimum bandwidth $\left(B_{f}\right)$ of $500 \mathrm{MHz}$. $k_{j}^{(v)}$ is carrier frequency during the $j$ th frame of $v$ th user which is pseudo random and takes any one of the frequency bands $0 \leq k_{j}^{(v)} \leq N_{f h}\left(N_{f h} B_{f}=B\right)$.

Fig. 1 shows the frequency and time hopping representation of the UWB signals for multiple users. Here the UWB pulse is transmitted in any one time slot occupying $T_{c}$ seconds and $B_{f}$ bandwidth.

For M-ary PPM, signal amplitude $A^{(v)}=1$ so that (1) can be written as

$$
s^{v}(t)=\sum_{j=-\infty}^{\infty} p\left(t-j T_{f}-c_{j}^{(v)} T_{c}-\delta_{d_{\left\lfloor j / N_{s}\right\rfloor}^{(v)}}\right) e^{-j 2 \pi k_{j}^{(v)} t}
$$

The received signal from multipath channel for each user is:

$$
r(t)=\sum_{v=1}^{N_{u}} s^{(v)}(t) \otimes g(t)+n(t)
$$

where, $n(t)$ is AWGN noise with power spectral density $N_{0} / 2, \otimes$ represents the convolution operator. $g(t)$ is unknown multipath channel given by:

$$
g(t)=\sum_{l=1}^{L} \alpha_{l}^{(v)} \delta\left(t-\tau_{l}\right)
$$

where, $\alpha_{l}^{(v)}$ is multipath gain co-efficient of $v$ th user in $l$ th path, and $\tau_{l}$ is the multipath delay.

Substituting $s^{(v)}$ in (3), $r(t)$ can be written as:

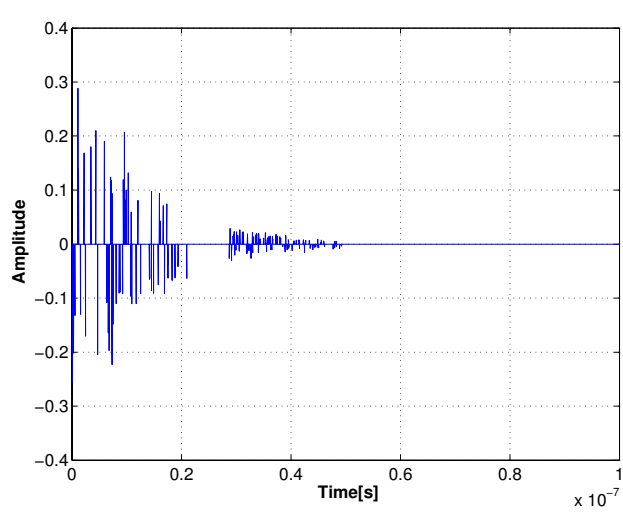

Figure 3. Channel impulse response of CM1

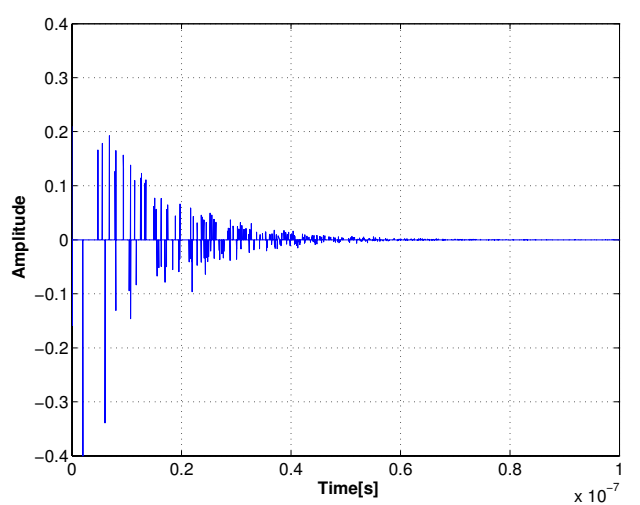

Figure 4. Channel impulse response of CM2

$$
\begin{gathered}
r(t)=\sum_{l=1}^{L} \sum_{v=1}^{N_{u}} \sum_{j=-\infty}^{\infty} p\left(t-j T_{f}-c_{j}^{(v)} T_{c}-\delta_{d_{\left\lfloor j / N_{s}\right\rfloor}^{(v)}}\right) \\
\alpha_{l}^{(v)} \delta\left(t-\tau_{l}\right)+n(t)
\end{gathered}
$$

PPM receiver uses Rake receiver followed by matched filter. Even though the number of users is more than one, an $\mathrm{M}$-ary correlation receiver is typically used for simplicity.

\section{MULTIPLE ACCESS INTERFERENCE AND ERROR PROBABILITY}

MAI is the factor limiting the performance and capacity of the system when more than one user is active. MAI can be modeled as a zero mean Gaussian random variable if number of users are large [13]. Assuming M-ary PPM signal to be orthogonal (i.e $\delta \geq T_{p}$ ) the MAI and error probability analysis is carried out as follows.

In order to evaluate MAI, we make the following assumptions:

(a) $s^{(v)}(t)$ for $v=1,2, \ldots, N_{u}$, where $N_{u}$ is the number of active users, and the noise $n(t)$ are assumed to be independent.

(b) The time hopping sequence $c_{j}^{(v)}$ and multipath time delay $\tau_{l}$ are assumed to be independent and identically distributed (iid) over the time interval $\left[0, T_{f}\right]$.

(c) The frequency hopping sequence $k_{j}^{(v)}$ is assumed to be independent and identically distributed over the 
frequency band $B$.

(d) Perfect synchronization is assumed at the receiver, i.e $\tau_{l}$ is known at the receiver.

Assume that $N_{s}=1$ and that the desired user corresponds to $v=1$.

Rake receiver consists of a M-ary correlator in each finger. User 1 basis function $h_{i}^{(1)}(t)$ is given by:

$$
h_{i}^{(1)}(t)=p\left(t-\delta_{i}\right), \quad i=1,2, \ldots, M .
$$

At sampling instant, $t=j T_{f}$, the output of $\mathrm{L}$ finger Rake filter $\hat{\boldsymbol{r}}_{i}, i=1, \ldots . ., M$ is

$$
\begin{aligned}
\hat{\mathbf{r}}_{i}= & \sum_{l=1}^{L} \sum_{j=n N_{s}+1}^{(n+1) N_{s}} \\
& \int_{(j-1) T_{f}}^{T_{f}} \mathbf{r}(t) h_{i}^{(1)}\left(t-j T_{f}-c_{i}^{(v)} T_{c}-\delta_{i}\right) d t
\end{aligned}
$$

Assuming PPM signal $\boldsymbol{s}_{m}$ is transmitted by user 1, (7) can be written as

$$
\hat{\boldsymbol{r}}_{i}= \begin{cases}L N_{s} \sqrt{E_{g}}+N_{M A I}+N & i=n \\ N_{M A I}+N & i \neq n\end{cases}
$$

where, $E_{g}$ is average signal energy. MAI component $N_{M A I}$ is

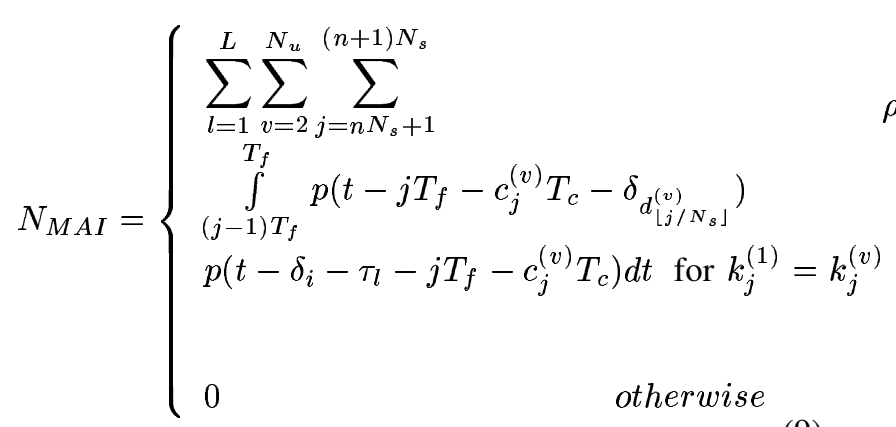

and AWGN component $\mathrm{N}$ is

$$
\begin{aligned}
N= & \sum_{l=1}^{L} \sum_{j=n N_{s}+1}^{(n+1) N_{s}} \\
& \int_{(j-1) T_{f}}^{T_{f}} n(t) p\left(t-\delta_{i}-\tau_{l}-j T_{f}-c_{j}^{(v)} T_{c}\right) d t
\end{aligned}
$$

By defining the autocorrelation function of $p(t)$ as

$$
\rho(\Delta)=\int_{0}^{T_{f}} p(t) p(t+\Delta) d t
$$

(9) can be written as

$$
N_{M A I}=\left\{\begin{array}{lc}
\sum_{l=1}^{L} \sum_{j=1}^{N_{s}} \sum_{v=2}^{N_{u}} \rho\left(\Delta_{j}^{(v)}\right) & k_{j}^{(1)}=k_{j}^{(v)} \\
0 & \text { otherwise }
\end{array}\right.
$$

where $\Delta_{j}^{(v)}=\left(c_{j}^{(1)}-c_{j}^{(v)}\right) T_{c}-\left(\delta_{i}^{(1)}-\delta_{d_{\left\lfloor j / N_{s}\right\rfloor}^{(v)}}\right)$ is the time difference between user 1 and user $v$.

Under the assumptions listed above, $\Delta$ can be modeled as a random variable which is uniformly distributed over $\left[-T_{f}, T_{f}\right]$. The MAI is modeled as a Gaussian random process for the multi-user environment [14]. With the Gaussian approximation we require the mean and variance of (8) to characterize the output of the cross correlators.

The AWGN component has zero mean and variance $N_{s} N_{0} / 2$ while the mean and variance of MAI are pulse waveform specific. The calculations are carried out considering the double differentiated Gaussian pulse as the transmitted pulse and all PPM signals are equally likely apriori. The double differentiated Gaussian pulse is defined as

$p(t)= \begin{cases}\sqrt{E_{g}}\left(1-\frac{4 \pi t^{2}}{\lambda T_{p}^{2}}\right) e^{-\frac{2 \pi t^{2}}{\lambda T_{p}^{2}}} & \frac{-T_{p}}{2} \leq t \leq \frac{T_{p}}{2} \\ 0 & \text { otherwise }\end{cases}$

where, $\lambda$ is the pulse shaping parameter. The autocorrelation of double differentiated Gaussian pulse is then given by

$\rho(\Delta)= \begin{cases}E_{g} \sqrt{\frac{\lambda T_{p}^{2}}{8}} e^{-\frac{\pi \Delta^{2}}{\lambda T_{p}^{2}}}(1.0606- & \\ \left.30.72 \frac{\Delta^{2}}{\lambda T_{p}^{2}}+11.6856 \frac{\Delta^{4}}{\lambda^{2} T_{p}^{4}}\right) & 0 \leq|\Delta| \leq \frac{T_{p}}{2} \\ 0 & \text { otherwise }\end{cases}$

$$
E[\rho(\Delta)]=\frac{1}{2 T_{f}} \int_{-T_{f}}^{T_{f}} \rho(\Delta) d \Delta=0
$$

The mean of $N_{M A I}$ can be calculated as

$$
\begin{gathered}
E\left[N_{M A I}\right]=E\left[\sum_{l=1}^{L} \sum_{j=1}^{N_{s}} \sum_{v=2}^{N_{u}} \rho\left(\Delta_{j}^{(v)}\right)\right] \\
E[M A I]=0
\end{gathered}
$$

and variance $N_{M A I}$ is

$$
\begin{aligned}
\operatorname{Var}\left[N_{M A I}\right]= & \operatorname{Var}\left[\sum_{l=1}^{L} \sum_{j=1}^{N_{s}} \sum_{v=2}^{N_{u}} \rho\left(\Delta_{j}^{(v)}\right)\right] \\
& =\sum_{l=1}^{L} \sum_{j=1}^{N_{s}} \sum_{v=2}^{N_{u}} E\left[\rho^{2}\left(\Delta_{j}^{(v)}\right)\right] \\
& =L N_{s} E_{g}\left(N_{u}-1\right) \frac{35 \sqrt{2} T_{p}}{192 T_{f}} \\
& \approx L N_{s} E_{g}\left(N_{u}-1\right) \frac{T_{p}}{4 T_{f}}
\end{aligned}
$$

Since $T_{f} / 2 T_{p}=N_{t h}$ (18) can be written as, 
TABLE I.

IEEE UWB CHANNEL MODEL PARAMETERS.

\begin{tabular}{lll} 
Parameter & CM1 & CM2 \\
& $\begin{array}{l}\text { LOS } \\
(0-4 \mathrm{~m})\end{array}$ & $\begin{array}{l}\text { NLOS } \\
(0-4 \mathrm{~m})\end{array}$ \\
\hline \hline Cluster arrival rate, $\Lambda(1 / \mathrm{ns})$ & 0.0233 & 0.4 \\
Ray arrival rate, $\lambda(1 / \mathrm{ns})$ & 2.5 & 0.5 \\
Cluster decay factor, $\Gamma$ & 7.1 & 5.5 \\
Ray decay factor, $\gamma$ & 4.3 & 6.7 \\
Std. dev. of cluster, $\sigma_{\zeta}(\mathrm{dB})$ & 3.3941 & 3.3941 \\
Std. dev. of ray, $\sigma_{\xi}(\mathrm{dB})$ & 3.3941 & 3.3941 \\
Std. dev. of total MP, $\sigma_{\mathrm{g}}(\mathrm{dB})$ & 3 & 3 \\
\hline
\end{tabular}

$$
\sigma_{M A I}^{2} \approx L N_{s} E_{g}\left(N_{u}-1\right) \frac{1}{8 N_{t h}}
$$

Now, due to frequency hopping, it can be easily shown that $\sigma_{M A I}^{2}$ will be reduced by a factor $N_{f h}$, and therefore (19) can be rewritten as

$$
\sigma_{M A I}^{2} \approx L N_{s} E_{g}\left(N_{u}-1\right) \frac{1}{8 N_{t h} N_{f h}}
$$

Note that $\sigma_{M A I}^{2}$ increases with $N_{s}, E_{g}$ and the number of users $N_{u}$, but decreases with the spread ratio $N_{t h}$ and $N_{f h}$

Using standard techniques [15] the average probability of error for a single user under multiple access interference for binary PPM is given by

$$
P_{M} \leq Q\left(L N_{s} \sqrt{\frac{E_{g}}{\sigma_{M A I}^{2}+\frac{N_{s} N_{0}}{2}}}\right)
$$

\section{Simulation RESUlts AND Discussion}

Simulations were carried out for synchronous user case. Bit error rate (BER) results are presented as a function of $E_{b} / N_{0}$ and number of users. The parameters considered for simulations are binary PPM with sampling frequency of $50 \mathrm{GHz}$, chip duration of 1 nanosecond, double differentiated Gaussian pulse of width 0.5 nanosecond and $\delta$ of 0.5 nanosecond. Frequency spectrum $3.1 \mathrm{GHz}-10.6$ $\mathrm{GHz}$ is divided into a maximum of 8 bands $\left(N_{f h}\right)$ each of $900 \mathrm{MHz}$ with a guard band of $42 \mathrm{MHz}$. Pseudo random

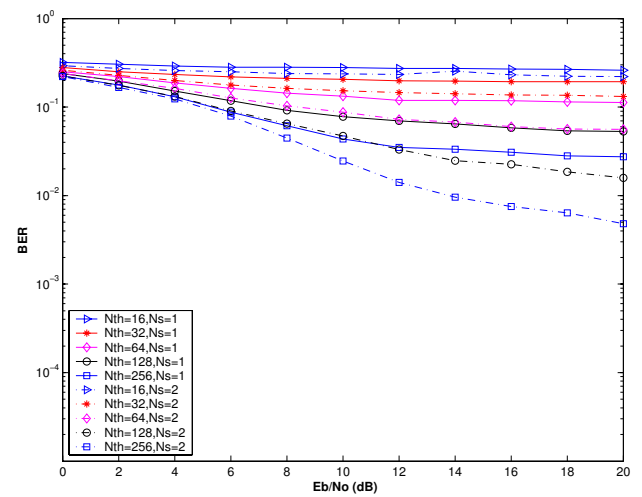

Figure 5. BER plot for Time hopping with $N_{u}=16$ in CM1

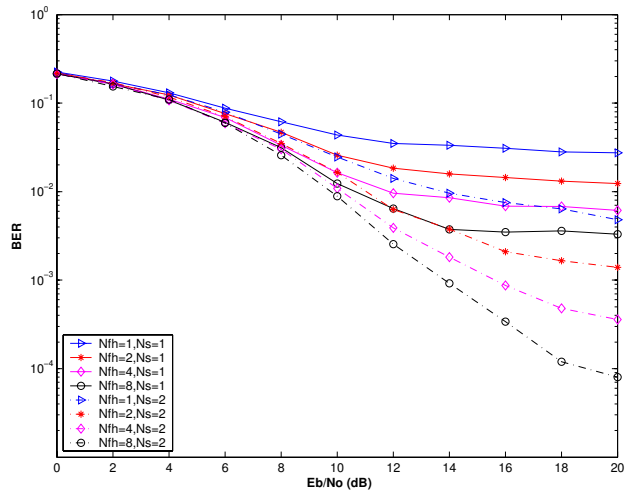

Figure 6. BER plot for Frequency-Time hopping with $N_{u}=16$ in CM1

frequency and time hopping codes of length 50000 is generated and assigned to each user.

Binary data is generated using uniform random number generator for each user and modulated using UWB pulse. Each user undergoes a different UWB channel. Channel models CM1 and CM2 from IEEE P802.15 [16] are used. Channel model parameters are listed in table I. Channel impulse response for CM1 and CM2 is shown in Figs. 3 and 4 respectively. Simulations are carried out for 1) fixed frequency hopping with varying time hops, 2) fixed number of time hops and varying frequency hops with and without repetitive coding $\left(N_{s}\right)$. Bit error probability is averaged over 100 channels for each user with 1000 bits/channel. The receiver implemented is a $\mathrm{L}$ finger Rake matched filter with Equal Gain Combiner. It is assumed that the frequency and time hopping sequence of the user of interest is known. To verify and investigate BER performance in a multiuser scenario, $N_{u}=16$ is considered.

Fig. 5 shows the BER performance of fixed frequency hopping $\left(N_{f h}=1\right)$ and varying time hopping PPM modulation in CM1 with $N_{s}=1$ and 2 . It is observed that by doubling the number of time hops BER performance improves by an average of $3 \mathrm{db}$ in lower $E_{b} / N_{0}$ range. However for $E_{b} / N_{0}>10 \mathrm{~dB}$, BER performance is limited by the MAI floor which is due to multi-access

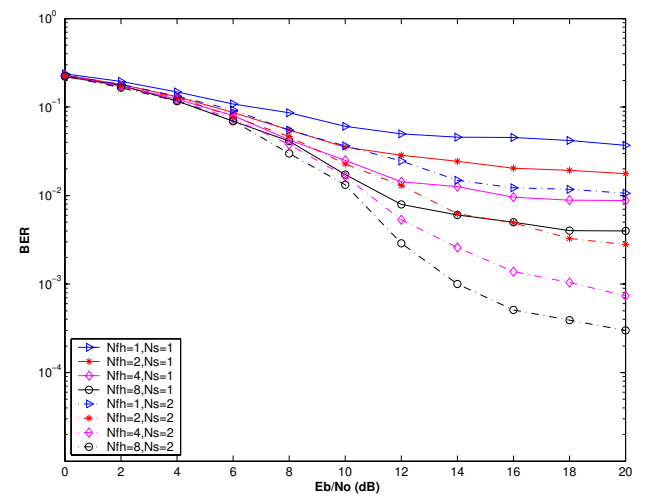

Figure 7. BER plot for Frequency-Time hopping with $N_{u}=16$ in CM2 


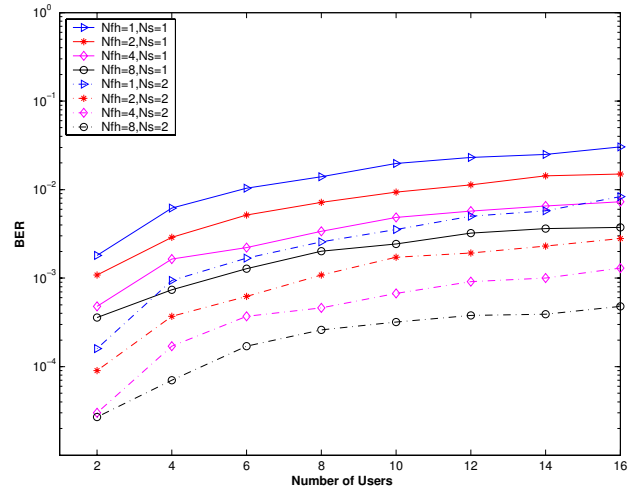

Figure 8. BER vs. Number of users in CM1

and intersymbol interference. It is also observed that with a repetition coding $\left(N_{s}=2\right)$ the BER performance is much better than without coding giving an advantage of $3 \mathrm{~dB}$. It is to be noted that increase in number of time hops reduces the data rate.

Fig. 6 shows the time and frequency hopping BER plots for fixed time hops $\left(N_{t h}=256\right)$ and varying frequency hops in CM1 with $N_{s}=1$ and 2. It is observed that introduction of frequency hopping along with time hopping gives an improvement of $3 \mathrm{~dB}$. Further, doubling the frequency hops gives an average improvement of $1 \mathrm{~dB}$ at BER of $10^{-2}$. This improved performance is due to a reduction in Multi-access and intersymbol interference. Once again it is observed that repetition coding gives substantial improvement for BER $<10^{-2}$.

Fig. 7 shows the time and frequency hopping BER plots for fixed time hops $\left(N_{t h}=256\right)$ and varying frequency hops in CM2 with $N_{s}=1$ and 2. It is observed that BER performance in CM2 is inferior than CM1 by $1 \mathrm{~dB}$. This is due to a large number of multipaths present in CM2, which results in increased intersymbol interference.

Fig. 8 and Fig. 9 show the BER vs. number of users performance for time hopping and time-frequency hopping for CM1 and CM2 respectively. It can be observed that the probability of error decreases with the introduction of frequency hopping as a result of which more number of

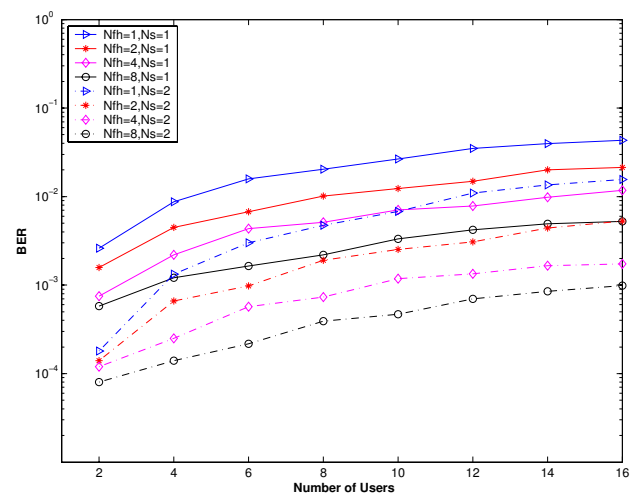

Figure 9. BER vs. Number of users in CM2 users is supported for a given BER. It is also observed that for a given BER, by doubling the number of frequency hops two more users can be accommodated.

\section{CONCLUSION}

In this paper we have proposed and analyzed bit error probability performance of frequency and time hoping PPM UWB multiple access communication in IEEE P802.15 multipath channel. We have derived an expression for the bit error probability for multi-user synchronous transmitter case. It is observed that introduction of frequency hopping along with time hopping improves BER performance by an average of $4 \mathrm{~dB}$. Further, doubling the number of frequency hops improves BER performance by $1 \mathrm{~dB}$. The proposed technique improves BER performance without reducing the data rate.

\section{REFERENCES}

[1] R. A. Scholtz, "Multiple Access with Time-hopping Impulse Modulation," in IEEE Military Communications Conference (MILCOM'93), vol. 2, Oct. 1993, pp. 447-450.

[2] http://www.ieee802.org/15/.

[3] M. Z. Win and R. A. Scholtz, "Ultra-wide bandwidth timehopping spread spectrum impulse radio for wireless multiple access communications," IEEE Trans., on Commun.,, vol. 48, no. 4, pp. 679-689, 2000.

[4] F. Ramirez-Mireles and R. A. Scholtz., "Multiple-Access Performance Limits with Time Hopping and Pulse Position Modulation," in IEEE Military Communications Conference (MILCOM'93), vol. 2, Oct. 1993, pp. 529-533.

[5] L. L. Yang and L. Hanzo, "Residue number system assisted fast frequency hopped synchronous ultra-wideband spreadspectrum multiple access: a design alternative to impulse radio," IEEE Journal on Sel. Areas of Commun., vol. 20, no. 9, pp. 1652-1663, 2002.

[6] J.R. Foerster, "The performance of direct-sequence spread ultra-wideband system in the presence of multipath, narrowband interference," in IEEE International Conference on Ultra Wideband Systems and Technologies (UWBST'02), vol. 3, 2002, pp. 87-91.

[7] C. M. Canadeo, M. A. Temple, R.O. Baldwin, and R.A. Raines, "Code selection for enhancing uwb multiple access communication performance using th-ppm and dsbpsk modulation," in IEEE International Conference on Wireless Commun., (WCNC'03)), vol. 1, 2003, pp. 678682.

[8] Ismail Guvenc and Huseyin Arslan, "Design and performance analysis of TH sequences for uwb-ir systems," in IEEE conf. Wireless Communications and Networking Conference (WCNC'04), vol. 2, 2004, pp. 21-25.

[9] F. Pingzhi, Moon Ho Lee and Daiyuan Pengi, "New family of hopping sequences for time/frequency hopping cdma systems," IEEE Trans. on Wireless Commun., vol. 4, pp. 2836-2842, 2005.

[10] Ebrahim Saberinia and Ahmed H. Tewfik, "Multi-user uwb-ofdm communications," in IEEE conf. Commun., Computers and Signal Processing (PACRIM 2003), vol. 1, 2003, pp. 127-230.

[11] Andreas F. Molisch and Jinyum Zhang, "Time hopping and frequency hopping in ultra wideband systems," in IEEE conf. Commun., Computers and Signal Processing (PACRIM 2003), vol. 2, 2003, pp. 541-544.

[12] A. W. Lam and D. V. Sarwate, "Time-hopping and frequency hopping multiple access packet communication," IEEE Trans. on Commun., vol. 38, no. 6, pp. 875-888, 1998. 
[13] F .Ramirez-Mireles and R.A. Scholtz, "Multiple access with time hopping and block waveform ppm modulation," in IEEE International Conference on Communications (ICC'98), vol. 2, 1998, pp. 775-779.

[14] G. Durisi and G. Romano, "On the validity of gaussian approximation to characterize the multiuser capacity of uwb th ppm," in IEEE Conference on Ultra Wideband Systems and Technologies (UWBST'02), vol. 1, 2002, pp. 157-161.

[15] John G. Proakis, Digital Communications, 4th ed. McGraw-Hill New York, USA, 2001

[16] A.F. Molisch, J.R. Foerster, and M. Pendergrass, "Channel models for ultra wideband personal area networks," IEEE Wireless Commun., Mag. , vol. 10, no. 6, pp. 14-21, 2003.

G.S. Biradar received his Bachelor of Engineering degree in Electronics and Communication form Gulbarga University Gulbarga in 1990 and M.Tech in Telecommunication Systems Engineering form IIT Kharagpur, India in 2002. From 1992 onwards he is working as a lecturer is P.D.A.College of Engineering Gulbarga. He worked on multiuser detectors for 3rd generation wireless communication. Currently he is working towards his doctoral thesis at IIT Bombay, India. His research interests are in wireless communication and adaptive signal processing.

S.N. Merchant received his B.Tech, M.Tech, and Ph.D degrees all from Department of Electrical Engineering IIT Bombay, India. He has more than 20 years of experience in teaching and research. He has made significant contributions in the filed of signal processing and its applications. His noteworthy contributions have been in solving state of art signal and image processing problems faced by Indian defence. His broad area of research interests are signal and image processing, multimedia communication, wireless sensor networks and wireless communications, and has published extensively in these areas. $\mathrm{He}$ has been a chief investigator for number of sponsored and consultancy projects. He has served as a consultant for both private industries and defence organization. $\mathrm{He}$ is a reviewer for many leading international and national journals and conferences. He was chair of local organization committee for IEEE International Conference on Computer Vision, 1998 (ICCV 98). $\mathrm{He}$ is fellow of IETE. He is a recipient of 10th IETE Prof. SVC Aiya Memorial award for his contribution in the field of detection and tracking.

U.B. Desai received the B. Tech. degree from Indian Institute of Technology, Kanpur, India, in 1974, the M.S. degree from the State University of New York, Buffalo, in 1976, and the Ph.D. degree from The Johns Hopkins University, Baltimore, U.S.A., in 1979, all in Electrical Engineering. From 1979 to 1984 he was an Assistant Professor in the Electrical Engineering Department at Washington State University, Pullman, WA, U.S.A., and an Associate Professor at the same place from 1984 to 1987. Since 1987 he has been a Professor in the Electrical Engineering Department at the Indian Institute of Technology - Bombay. He has held Visiting Associate Professor's position at Arizona State University, Purdue University, and Stanford University. $\mathrm{He}$ was a visiting Professor at EPFL, Lausanne during the summer of 2002. From July 2002 to June 2004 he was the Director of HP-IITM R and D Lab. at IIT-Madras. His research interest is in wireless communication, wireless sensor networks and statistical signal processing. He is the Editor of the book "Modeling and Applications of Stochastic Processes" (Kluwer
Academic Press, Boston, U.S.A. 1986). He is also a co-author of two books "A Bayesian Approach to Image Interpretation" and "Multifractal based Network Modeling", both from Kluwer Academic Press. Dr. Desai is a senior member of IEEE, a Fellow of INSA (Indian National Science Academy), Fellow of Indian National Academy of Engineering (INAE). He is on the Executive Committee (EC) for the All India Council of Technical Education (AICTE). He was an associate editor of IEEE Transactions on Image Processing from Jan 1999 to Dec.2001. $\mathrm{He}$ is Vice-President of the Indian Unit for Pattern Recognition and Artificial Intelligence. He is on the Technology Advisory Board of Microsoft Research Lab. India. He was associate Vice Chair for PHY/MAC for IEEE International Conference for Wireless Communication and Networking (WCNC) 2005, TPC Chair for WPMC 2007, and TPC Co-Chair for COMSWARE 2008. He is the Chair for IEEE Bombay Section. He is also on the Visitation Panel for University of Ghana. 